\title{
Some algorithmic improvements for the containment problem of conjunctive queries with negation
}

\author{
Michel Leclère and Marie-Laure Mugnier \\ LIRMM, Université de Montpellier, \\ 161, rue Ada, F-34392 Montpellier cedex - France \\ \{leclere,mugnier\}@lirmm.fr
}

\begin{abstract}
Query containment is a fundamental problem of databases. Given two queries $q_{1}$ and $q_{2}$, it asks whether the set of answers to $q_{1}$ is included in the set of answers to $q_{2}$ for any database. In this paper, we investigate this problem for conjunctive queries with negated subgoals. We use graph homomorphism as the core notion, which leads us to extend the results presented in [U1197] and [WL03]. First, we exhibit sufficient (but not necessary) conditions for query containment based on special subgraphs of $q_{2}$, which generalize that proposed in [WL03]. As a corollary, we obtain a case where the time complexity of the problem decreases. From a practical viewpoint, these properties can be exploited in algorithms, as shown in the paper. Second, we propose an algorithm based on the exploration of a space of graphs, which improves existing algorithms.
\end{abstract}

\section{Introduction}

In this paper, we investigate the problem of deciding on query containment for conjunctive queries with negated subgoals (but without inequalities). Query containment checking is one of the fundamental problems of databases. A query $q_{1}$ is said to be contained in a query $q_{2}$ (notation $q_{1} \sqsubseteq q_{2}$ ) if for any database instance the set of answers to $q_{1}$ is included in the set of answers to $q_{2}$. Algorithms based on query containment can be used to solve various problems, such as query evaluation and optimization [CM77] [ASU79], rewriting queries using views [Hal01], detecting independance of queries from database updates [LS93], etc. However, the problem is undecidable for general queries expressed as Datalog programs.

Positive conjunctive queries are a class of frequently used queries which have been investigated since the early days of databases [CM77,U1189]. Their expressive power is equivalent to the select-join-project queries of relational algebra. Checking containment of positive conjunctive queries is an NP-complete problem. It can be solved by testing the existence of a query homomorphism from $q_{2}$ to $q_{1}$, which maps $q_{2}$ to $q_{1}$ by substituting its variables by terms (constants or variables) in $q_{2}$.

Example 1. Let $q_{1}=\operatorname{ans}(x, y) \leftarrow r(x, y), r(y, x), p(x, x), s(y)$ and $q_{2}=\operatorname{ans}(u, v) \leftarrow$ $r(u, v), r(v, w), p(u, w)$ be two conjunctive queries. There is one query homomorphism from $q_{2}$ to $q_{1}$, which is $h=\{u \rightarrow x, v \rightarrow y, w \rightarrow x\}$. Check that $h\left(q_{2}\right)$ has the same head as $q_{1}$ and its body is a part of $q_{1}$ 's body. This proves that $q_{1} \sqsubseteq q_{2}$. 
This problem can also be recast as a query evaluation problem by considering the canonical database associated with a query. Roughly, this database $D^{q}$ is obtained from a query $q$ by "freezing" its variables, that is considering them as new elements of the schema domain. Then query containment can be reformulated as evaluating $q_{2}$ on $D^{q_{1}}$ and checking that the set of answers contains the head of $q_{1}$ [CM77].

When conjunctive queries are extended to negated subgoals, query containment becomes $\Pi_{P}^{2}$-complete $\left(\Pi_{P}^{2}\right.$ is the class $\left.(c o-N P)^{N P}\right)$. To our best knowledge, only two proposals about algorithms deciding on query containment for this class of queries can be found in the literature. We outline the main points of these proposals here, and will go into further detail later. In [U1197], Ullman gives the scheme of an algorithm (adapted from a uniform equivalence checking method for Datalog programs [LS93]). This scheme involves generating an exponential number (in the size of $q_{1}$ ) of databases representative of $q_{1}$ and evaluating $q_{2}$ on these databases. This set of databases can be seen as a generalization of the canonical database of the positive case. A database that does not yield the head of $q_{1}$ as an answer to $q_{2}$ is a counter-example to the containment.

Example 2. Let $q_{1}=\operatorname{ans}() \leftarrow r(x, y), s(y, z), p(t), p(z), \neg r(z, t)$ and $q_{2}=$ ans ()$\leftarrow$ $r(u, v), p(w), \neg r(v, w)$. As ans () has no argument, these queries represent boolean queries. It holds that $q_{1} \sqsubseteq q_{2}$, as will be shown later. In a first step, Ullman's scheme builds the 15 partitions on $\{x, y, z, t\}$, which can be seen as all ways of mapping the variables in $q_{1}$ to database values. Each partition yields a database by substituting in $q_{1}$ the same value to each set of variables and taking the positive part of the query obtained. For instance, the partition $\{\{x, y\},\{z, t\}\}$ yields the database $\{r(0,0), s(0,1), p(1)\}$. If a database does not make the body of $q_{1}$ true, as the database obtained from the partition $\{\{x, z\},\{y, t\}\}$, it is eliminated. In a second step, for each database $D$, all its extensions obtained by adding tuples using the values and the relation symbols in $D$, and that still make the body of $q_{1}$ true, are considered and it is checked that they yield the substituted head of $q_{1}$ as an answer to $q_{2}$. For instance, for $D=\{r(0,0), s(0,1), p(1)\}$, all extensions with tuples $r(0,1), r(1,0), s(0,0), s(1,0), s(1,1)$ and $p(0)$ are considered.

In the general case, if $v$ is the number of variables in $q_{1}$, a number of databases exponential in $v$ are generated in the first step, then, for each generated database $D_{i}$, $2^{\left(\left(\sum_{r \in R} n_{i}^{a r i t y(r)}\right)-t\right)}$ representative databases have to be checked, where $R$ is the set of relation symbols appearing in $q_{1}, n_{i}$ is the number of terms in $D_{i}$ and $t$ is the number of tuples in $q_{1}$.

In [WL03], Wei and Lausen exhibit a necessary but not sufficient condition for containment of safe queries (which are queries in which all variables appear in positive subgoals): if $q_{1}$ is contained in $q_{2}$ then there must be a query homomorphism (say $h$ ) from the positive part of $q_{2}$ (say $q_{2}^{+}$) to the positive part of $q_{1}$ (say $q_{1}^{+}$), that does not "contradict" the negative subgoals of $q_{2}$ (i.e. for all negative subgoals $\neg p(u)$ in $q_{2}, q_{1}$ does not contain the positive subgoal $p(h(u)))$. This property is central to the proposed algorithm. It yields a heuristic for the generation of representative databases, with the aim of concluding sooner from partial representative databases. To check that $q_{1} \sqsubseteq q_{2}$, the algorithm tries to find a query homomorphism (without contradiction) $h$ from $q_{2}^{+}$to $q_{1}^{+}$, such that for each negative literal $\neg p(u)$ in $q_{2}$, either $\neg p(h(u))$ is in $q_{1}$ or the query $q_{1}^{\prime}$ built from $q_{1}$ by adding $p(h(u))$ is such that $q_{1}^{\prime} \sqsubseteq q_{2}$. 
Let us outline this algorithm in example 2. There are 2 homomorphisms from $q_{2}^{+}=$ ans ()$\leftarrow r(u, v), p(w)$ to $q_{1}^{+}: h_{1}=\{u \rightarrow x, v \rightarrow y, w \rightarrow z\}$ and $h_{2}=\{u \rightarrow x, v \rightarrow$ $y, w \rightarrow t\}$. Both homomorphisms do not contradict any negative subgoal in $q_{2}$. Let us consider $h_{1}$ and the negative literal $\neg r(v, w)$ in $q_{2}$. The idea is that any database answering $q_{1}$ that does not contain $r(y, z)$ also answers $q_{2}$, thus databases containing $r(y, z)$ have to be checked. $r(y, z)$ is added to $q_{1}$, yielding $q_{1}^{\prime}$. There are four query homomorphisms from $q_{2}^{+}$to $q_{1}^{\prime+}$. If it can be concluded that $q_{1}^{\prime} \sqsubseteq q_{2}$, then $q_{1} \sqsubseteq q_{2}$. Otherwise, the homomorphism $h_{2}$ has to be considered.

Example 3. (ex. 1.2 in [WL03]) Let $q_{1}=\operatorname{ans}(x, y) \leftarrow r(x, y), r(y, z), \neg r(x, z)$ and $q_{2}=\operatorname{ans}(u, w) \leftarrow r(u, v), r(v, w), \neg s(w, w)$. There is one query homomorphism, $h=\{u \rightarrow x, v \rightarrow y, w \rightarrow z\}$, from $q_{2}^{+}=\operatorname{ans}(u, w) \leftarrow r(u, v), r(v, w)$ to $q_{1}^{+} . h$ does not contradict the negative subgoal of $q_{2}$. Then, $q_{1}^{\prime}$ is generated from $q_{1}$ by adding $s(z, z)$. Again $h$ is the sole homomorphism from $q_{2}^{+}$to $q_{1}^{\prime+}$ but it contradicts $\neg s(w, w)$. Thus, $q_{1}^{\prime} \nsubseteq q_{2}$ and as there is no other homomorphism from $q_{2}^{+}$to $q_{1}^{+}$it is concluded that $q_{1} \not q_{2}$.

Contribution. In this paper, we consider homomorphism as a core notion, where a homomorphism is not restricted to the positive parts of queries as in previous proposals, but extended to whole queries. For this, we propose to view the queries as labeled graphs, called polarized graphs. More specifically, a query is represented as a bipartite graph, with two kinds of nodes: relation nodes and terms nodes ${ }^{1}$. Each term of the query becomes a term node, labeled by $\star$ if it is a variable (it can be seen as a "blank node") otherwise by the constant itself. A positive (resp. negative) literal with relation symbol $r$ becomes a relation node labeled by $+r$ (resp. $-r$ ) and it is linked to the nodes assigned to its terms. The numbers on edges correspond to the position of each term in the literal. See Figure 1, which displays the queries in example 2.

Basically, a homomorphism from an algebraic structure to another maps the elements of the first structure to elements of the second structure while preserving the relations between elements. A homomorphism $h$ from a graph $G_{2}$ to a graph $G_{1}$ is a mapping from the nodes of $G_{2}$ to the nodes of $G_{1}$, which preserves edges, that is if $x y$ is an edge of $G_{2}$ then $h(x) h(y)$ is an edge of $G_{1}$. Since our graphs are labeled, there are additional conditions on labels: a relation node is mapped to a node with the same label; a term node can be mapped to any term node if it is labeled by a $\star$, otherwise it is mapped to a node with the same constant. Numbers on edges are preserved.

Graph homomorphism yields another perspective on queries, as it naturally considers positive and negative occurrences of relations in the same way; moreover, it is defined on subgraphs that do not necessarily correspond to a query, which is convenient for our study. However, let us point out that all definitions and results in this paper can be expressed using the classical vocabulary on queries. In what follows, the term homomorphism can be understood as "query homomorphism extended to negative subgoals" or "graph homomorphism".

A first property, extending the central property in [WL03], is that the existence of a homomorphism from $q_{2}$ to $q_{1}$ is a sufficient condition for containment.

\footnotetext{
${ }^{1}$ Queries have often been considered as hypergraphs. The graphs we consider can be seen as the incidence bipartite graphs of these hypergraphs.
} 


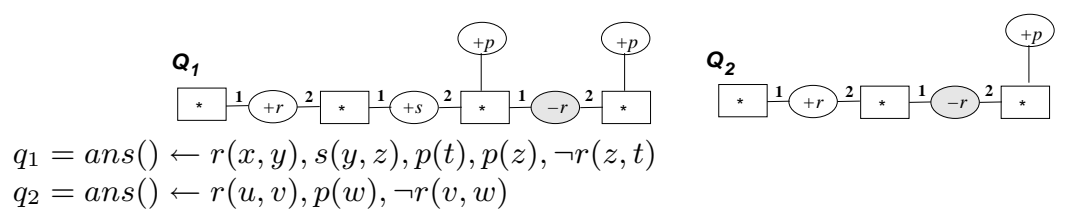

Fig. 1. Queries as graphs

Example 4. Let $q_{1}=\operatorname{ans}(y, z) \leftarrow r(x, z), r(y, z), \neg r(x, y)$ and $q_{2}=a n s(u, v) \leftarrow$ $r(u, v), r(w, e) \neg r(w, u)$. There is a homomorphism, say $h$, from $q_{2}$ to $q_{1}$, thus $q_{1} \sqsubseteq q_{2}$. $h=\{w \rightarrow x, u \rightarrow y, v \rightarrow z, e \rightarrow z\}$.

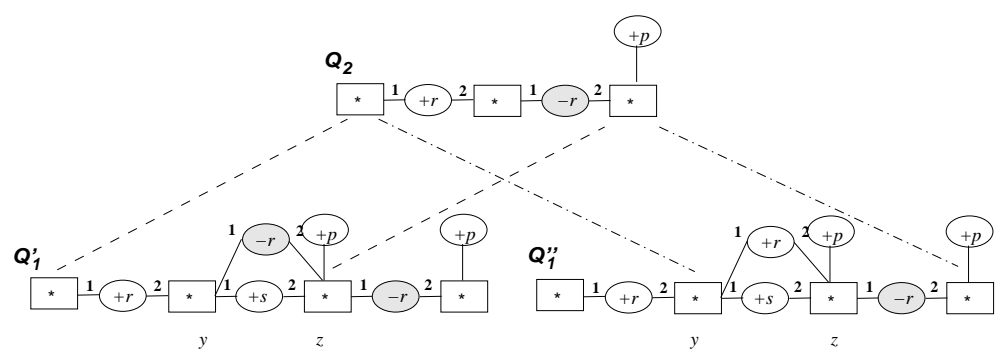

Fig. 2. Graph homomorphisms from $Q_{2}$ to $Q^{\prime}{ }_{1}$ and $Q^{\prime \prime}{ }_{1}$

The existence of a homomorphism is not a necessary condition, as can be seen in example 2 (pictured in Figure 1): $q_{1} \sqsubseteq q_{2}$ but there is no homomorphism from $q_{2}$ to $q_{1}$. However, $q_{1}$ can be equivalently rewritten as the union of two queries: one obtained by adding $\neg r(y, z)$, the other by adding $r(y, z)$. These queries are shown in Figure 2. As there is a homomorphism from $q_{2}$ to both queries, we conclude that $q_{1} \sqsubseteq q_{2}$.

More generally, instead of considering representative databases, we rewrite $q_{1}$ into more and more specific queries. We are lead to consider a space of graphs (or queries) partially ordered by inclusion, with greatest element $q_{1}$ and least elements the "complete" graphs, obtained from $q_{1}$ by adding as many literals as possible. A brute-force algorithm generates all complete graphs and check that there is a homomorphism from $q_{2}$ to each of them.

Roughly, Ullman's scheme can be seen as generating all complete graphs from $q_{1}$. We should point out, however, that the first step in computing all partitions on $q_{1}$ terms is not necessary, i.e. the discrete partition is sufficient. The set of representative databases generated from the discrete partition is the set of complete graphs. Although it is not claimed in [Ul197], Ullman's algorithm is in fact able to process queries with inequalities (see section 2.4).

This framework being settled, we focus on two points. First, we search for cases where the problem is simpler. We study special subgraphs of $q_{2}$ that necessarily map to $q_{1}$ (theorem 2 and 3); $q_{2}^{+}$is a specific case. As a corollary, when the whole $q_{2}$ satisfies 
one of these conditions, the containment problem becomes equivalent to homomorphism checking, thus its time complexity falls into NP (property 8). From a practical viewpoint, these properties can be exploited in algorithms, including Wei and Lausen's algorithm. They can be used in a preprocessing phase (to limit the number of representative databases or to conclude before entering the generation phase) or to guide the construction of representative databases. Second, we propose an algorithm based on exploration of the graph space. This algorithm is simple to describe and implement. Its correctness is directly given by the exhibited properties (theorem 4) and its complexity is analyzed (property 10). We compare this algorithm to Wei and Lausen's algorithm, which can be seen as exploring the same space of graphs but in a radically different way. In particular, our algorithm requires a space polynomial in the size of the initial queries (provided that the maximal arity of a relation is bounded by a constant), which is not the case for Wei and Lausen's algorithm.

The paper is organized as follows. The next section introduces the graph framework and reformulates the query containment problem in terms of graph homomorphism. It ends with a brute-force algorithm, whose complexity is compared to that of Ullman's scheme. Section 3 is devoted to necessary or sufficient conditions for containment. Section 4 presents our algorithm based on space exploration and compares it to Wei and Lausen's algorithm.

\section{Preliminary considerations}

We first recall basic definitions and results on databases. Then we introduce the graph framework, which leads us to recast CQC as a conjunction of homomorphism tests. We end with a brute-force algorithm, that we compare with Ullman's scheme.

\subsection{Basic database notions}

A database schema $S=(R$, dom $)$ includes a finite set of relations $R$ and a countably infinite set of constants dom. Each relation has an arity (not equal to zero) defining the number of its arguments. A database instance $D$ (or simply a database) over $S$ maps each $k$-ary relation $r_{i}$ of $R$ to a finite subset of $d_{o m}^{k}$ (denoted $D\left(r_{i}\right)$ ). A conjunctive query (with negation) is of form:

$q=\operatorname{ans}(u) \leftarrow r_{1}\left(u_{1}\right), \ldots r_{n}\left(u_{n}\right), \neg s_{1}\left(v_{1}\right), \ldots \neg s_{m}\left(v_{m}\right) n \geq 0, m \geq 0, n+m \geq 1$ where $r_{1} \ldots r_{n}, s_{1} \ldots s_{m}$ are relations, ans is a special relation not belonging to $R, u$ and $u_{1} \ldots u_{n}, v_{1} \ldots v_{m}$ are tuples of terms (variables or constants of $d o m$ ), and each variable of $u$ occurs at least once in the body of the rule. Without loss of generality, we assume that the same literal does not appear twice in the body of the rule. A positive query is a query without negative literals ( $m=0$, thus $n \geq 1$ ). A query is safe if each variable occurring in a negative literal also occurs in a positive one. A query is inconsistent if it contains two opposite literals (i.e. $\exists i, j 1 \leq i \leq n, 1 \leq j \leq m$ such that $\left.r_{i}\left(u_{i}\right)=s_{j}\left(v_{j}\right)\right)$, otherwise it is consistent.

Given a query $q=\operatorname{ans}(u) \leftarrow r_{1}\left(u_{1}\right), \ldots r_{n}\left(u_{n}\right), \neg s_{1}\left(v_{1}\right), \ldots \neg s_{m}\left(v_{m}\right)$ and a database $D$ on $S, q(D)$ denotes the set of answers to $q$ in $D ; q(D)$ is the set of tuples 
$\mu(u)$ where $\mu$ is a substitution of the variables in $q$ by constants in $d o m$ such that for any $i$ in $\{1, \ldots, n\}, \mu\left(u_{i}\right) \in D\left(r_{i}\right)$ and for any $j$ in $\{1, \ldots, m\}, \mu\left(v_{j}\right) \notin D\left(s_{j}\right)$. We also call $\mu$ a substitution from $q$ to $D$. When the arity of ans is $0, q(D)$ is the set $\{()\}$ if there is such a substitution $\mu$, otherwise it is $\emptyset$. If $q(D)$ is not empty, $D$ is said to answer the query.

A query $q_{1}$ is said to be contained in a query $q_{2}$, noted $q_{1} \sqsubseteq q_{2}$, if for any database $D, q_{1}(D) \subseteq q_{2}(D)$. The conjunctive query containment problem (CQC) takes as input two conjunctive queries $q_{1}$ and $q_{2}$ and asks whether $q_{1} \sqsubseteq q_{2}$. When $q_{1}$ and $q_{2}$ are positive, it can be reformulated as a query homomorphism problem, where a homomorphism is defined as follows: a query homomorphism from $q=$ ans $(u) \leftarrow$ $r_{1}\left(u_{1}\right), \ldots r_{n}\left(u_{n}\right)$ to $q^{\prime}=\operatorname{ans}\left(u^{\prime}\right) \leftarrow r_{1}^{\prime}\left(u_{1}^{\prime}\right), \ldots r_{n^{\prime}}^{\prime}\left(u_{n^{\prime}}^{\prime}\right)$ is a substitution $\theta$ of the variables in $q$ by terms (variables or constants) in $q^{\prime}$ such that $\theta(u)=u^{\prime}$ (thus $u$ and $u^{\prime}$ have the same size) and for any $i$ in $\{1, \ldots, n\}$, there is $j$ in $\left\{1, \ldots, n^{\prime}\right\}$ such that $\theta\left(r_{i}\left(u_{i}\right)\right)=r_{j}^{\prime}\left(u_{j}^{\prime}\right)$. The query homomorphism theorem proves that, given two positive queries $q_{1}$ and $q_{2}, q_{1} \sqsubseteq q_{2}$ iff there is a query homomorphism from $q_{2}$ to $q_{1}$.

\subsection{CQC and homomorphism}

As explained in the introduction, it is convenient to see a query as a bipartite labeled graph, that we call a polarized graph $(\mathrm{PG})^{2}$. The mappings between graph and database notions used in this paper are immediate. To represent heads of queries, we use special relations $a n s_{i}$ for each possible arity $i$, possibly 0 (which corresponds to boolean queries). Then the head of a query (say ans $\left(t_{1} \ldots t_{k}\right)$ ) is mapped to a positive relation node with label $a n s_{k}$ and with $i$-th neighbor the node assigned to $t_{i}$. We usually omit $a n s_{0}$ in drawings (f.i. Figure 1: there is an implicit isolated relation node labeled $a n s_{0}$ in each graph). It is easily checked that a graph homomorphism from a graph representing a query to another is equivalent to a query homomorphism extended to negative subgoals from the first query to the second (the above definition of a query homomorphism can be used without change if we consider that $r_{i}$ and $r_{j}^{\prime}$ represent possibly negated relation). That is why we use the same term homomorphism for both notions. If there is a homomorphism from $q_{2}$ to $q_{1}$, we say that $q_{2}$ can be mapped to $q_{1}$. We will keep the term literal and its notation $p(u)$ or $\neg p(u)$, where $u$ is a sequence of terms, to denote a subgraph induced by a relation node and its neighbors.

For positive conjunctive queries $q_{1}$ and $q_{2}, q_{1} \sqsubseteq q_{2}$ iff there is a homomorphism from $q_{2}$ to $q_{1}$. For conjunctive queries with negation, one part of this property still holds:

Property 1. Given conjunctive queries $q_{1}$ and $q_{2}$, if there is a homomorphism from $q_{2}$ to $q_{1}$ then $q_{1} \sqsubseteq q_{2}$.

For the other direction, we assume that $q_{1}$ and $q_{2}$ are consistent. This assumption will be made in the sequel of the paper. Even if $q_{1}$ and $q_{2}$ are consistent, we might have $q_{1} \sqsubseteq q_{2}$ and no homomorphism from $q_{2}$ to $q_{1}$, as illustrated by Figures 1 and 2 .

\footnotetext{
${ }^{2}$ For space limitation reasons, we do not provide here precise definitions concerning PGs. These graphs are a simplification of graphs used in a knowledge representation context, see [Ker01,ML06].
} 
Definition 1. A consistent query (or a $P G$ ) $q$ is complete w.r.t. a set of relations $R$, if for each relation $r$ in $R$ with arity $k$, for each $k$-tuple of terms $u$ in $q$, not necessarily distinct, $q$ contains $r(u)$ or $\neg r(u)$.

A complete query is obtained from a query $q$ by repeatedly adding positive and negative literals (on terms already present in $q$ ), as long as it does not yield a redundancy or an inconsistency. CQC can be expressed as the conjunction of homomorphism checking problems: one for each complete query generated from $q_{1}$.

Property 2. Given two conjunctive queries $q_{1}$ and $q_{2}$ (with $q_{1}$ being consistent), $q_{1} \sqsubseteq q_{2}$ iff for each complete query $q_{1}^{c}$ generated from $q_{1}$, there is a homomorphism from $q_{2}$ to $q_{1}^{c}$

Note that $q_{2}$ can be considered as a connected graph: indeed, if $q_{2}$ is not connected, a homomorphism from $q_{2}$ to $q_{1}$ is given by a set of homomorphisms from each connected component of $q_{2}$ to $q_{1}$, and reciprocally.

\subsection{A brute force algorithm for CQC}

Property 2 yields a brute force algorithm (cf. algorithm 1) for CQC.

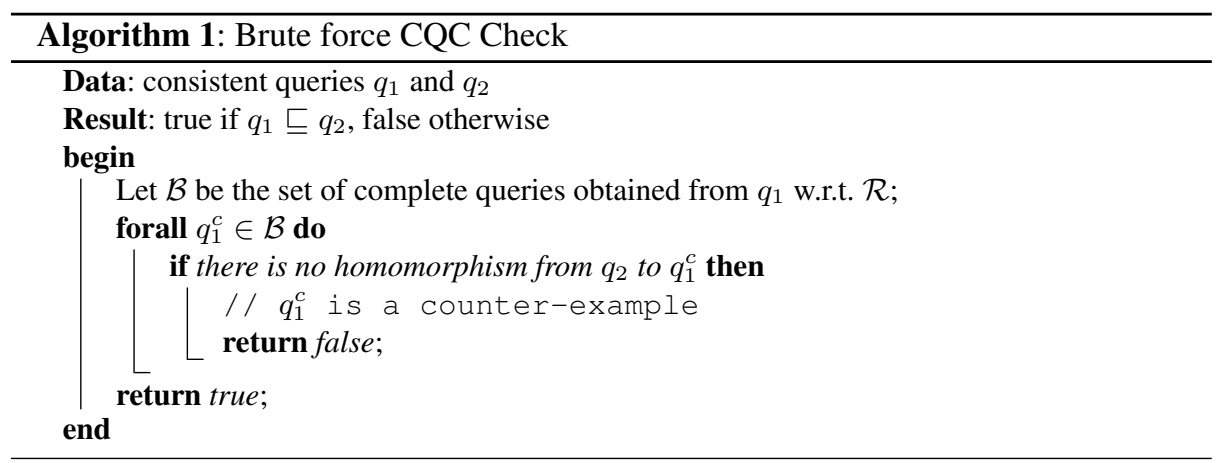

Property 3. The time complexity of Algorithm 1 is in $\mathcal{O}\left(2^{\left(n_{1}\right)^{k} \times|\mathcal{R}|} \times \operatorname{hom}\left(q_{2}, q_{1}^{c}\right)\right)$, where $n_{1}$ is the number of terms in $q_{1}, k$ is the maximum arity of a relation, $\mathcal{R}$ is the set of considered relations and $\operatorname{hom}\left(q_{2}, q_{1}^{c}\right)$ is the complexity of checking the existence of a homomorphism from $q_{2}$ to $q_{1}^{c}$.

Its space complexity is in $\mathcal{O}\left(\max \left(\operatorname{size}\left(q_{2}\right)\right.\right.$, size $\left.\left.\left(q_{1}\right),\left(n_{1}\right)^{k} \times|\mathcal{R}|\right)\right)$.

Homomorphism checking is NP-complete (but polynomial as soon as $q_{2}$ has a treelike structure). A brute force algorithm solving this problem for $q_{2}$ and $q_{1}^{c}$ has a time complexity in $\mathcal{O}\left(\min \left(n_{1}^{v_{2}}, r_{1}^{r_{2}}\right)\right)$, where $n_{1}$ is the number of term nodes in $q_{1}^{c}, v_{2}$ is the number of variable nodes in $q_{2}, r_{1}$ and $r_{2}$ are the number of literals in $q_{1}^{c}$ and $q_{2}$ resp. For comparison with other algorithms, it should be noted that the space complexity of 
Algorithm 1 is not exponential in the size of $q_{1}$ or $q_{2}$ but only in the maximum arity of a relation in $\mathcal{R}$. Indeed, as completions can be generated one by one, the space complexity corresponds to the size of one $q_{1}^{c}$.

\subsection{Relationships with Ullman's scheme}

Ullman's scheme involves the two following steps:

1. Consider all partitions of the variables in $q_{1}$. Build a canonical database from each partition as follows: first assign a distinct constant to each set of the partition, then substitute in $q_{1}$ each variable by the constant assigned to its set; let $q_{1}^{\prime}$ be the substituted query; the canonical database is composed of the positive literals of $q_{1}^{\prime}$ body. We obtain $D_{1} \ldots D_{k}$ canonical databases if $k$ is the number of partitions. Eliminate the $D_{i}$ which do not make the body of $q_{1}$ true, (i.e. the body of $q_{1}^{\prime}$ is inconsistent).

2. For each remaining $D_{i}$, test whether for each database $D^{\prime} i$ obtained from $D_{i}$ by adding tuples on the same symbol set as $D_{i}$, and without contradicting negative subgoals of $q_{1}$, it holds that $q_{2}\left(D_{i}^{\prime}\right)$ includes the head of $q_{1}^{\prime}$. If all $D_{i}$ satisfy the test, then $q_{1} \sqsubseteq q_{2}$, otherwise not.

This scheme can be reformulated as follows in our framework:

1. Build all consistent queries $D_{i}$ obtainable from $q_{1}$ by merging some variables.

2. The test is satisfied iff $q_{2}$ can be mapped to all complete queries obtainable from these $D_{i}$.

From property 2 , it is clear that step 1 is useless. Indeed, there is a homomorphism from $q_{1}$ to each $D_{i}, 1 \leq i \leq k, q_{1}$ being identical to the $D_{i}$ obtained with the discrete partition, say $D_{1}$. For a given $D_{i}^{\prime}$ there is a $D_{1}^{\prime}$ with a homomorphism from $D_{1}^{\prime}$ to $D_{i}^{\prime}$ induced by the partition on the variables in $q_{1}$ yielding $D_{i}$. It is thus sufficient to test all $D_{1}^{\prime}$, i.e. all complete queries obtainable from $q_{1}$. This observation leads to an important reduction in the number of tested databases/queries: if $v$ is the number of variables in $q_{1}$, step 1 builds a number of databases $D_{i}$ exponential in $v$ (from which only consistent ones are kept) and each remaining $D_{i}$ leads in turn to an exponential test (see Algorithm 1).

Step 1 would be necessary if the queries would contain inequalities as in [LS93]. However in [U1197] and further papers dealing with queries without inequalities, it seems that the uselessness of step 1 had not be noticed.

\section{Necessary / sufficient conditions for containment}

This section studies conditions that are necessary or sufficient for containment. These properties can be used as filtering properties leading to conclude before entering the generation phase. They can also be used to reduce the number of graphs generated either because they eliminate relations that are not needed in the completion or because they guide the incremental generation of complete graphs (see the next section). Besides their practical algorithmic interest, they also yield particular cases where the theoretical complexity of CQC decreases. 


\subsection{Immediate properties on labels}

Let us begin by considering the node labels. An immediate observation is that if a constant or a relation label (that is a relation with a given polarity) in $q_{2}$ does not appear in $q_{1}$, then $q_{1} \nsubseteq q_{2}$. A second observation is that relations that do not appear in both $q_{1}$ and $q_{2}$ are not needed in the completion of $q_{1}$. The next property takes the polarity of their occurrences into account.

Property 4. If $r$ is a relation that does not have both positive and negative occurrences in $q_{2}$, then $r$ is not needed in the completion of $q_{1}$ (i.e. $q_{1} \sqsubseteq q_{2}$ iff $q_{2}$ can be mapped to each completion of $q_{1}$ built without considering $r$ ).

Proof. $(\Leftarrow)$ If $q_{2}$ can be mapped to each complete query without considering $r$ then $q_{1} \sqsubseteq q_{2}$. Indeed, let $q_{1}^{c}$ be any complete query built from $q_{1}$. Let $q_{1}^{c-\{r\}}$ be obtained from $q_{1}^{c}$ by removing all literals, occurrences of $r$, that do not belong to $q_{1}$. There is a natural homomorphism, say $h_{1}$ from $q_{1}^{c-\{r\}}$ to $q_{1}^{c}$. By hypothesis there is a homomorphism, say $h$, from $q_{2}$ to $q_{1}^{c-\{r\}}$. The composition of these homomorphisms $h_{1} \circ h$ is a homomorphism from $q_{2}$ to $q_{1}^{c}$.

$\left(\Rightarrow\right.$ ) Let $q_{1} \sqsubseteq q_{2}$. Assume that $q_{1}^{c-\{r\}}$ is a completion (without considering $r$ ) of $q_{1}$ such that there is no homomorphism from $q_{2}$ to $q_{1}^{c-\{r\}}$. We show that this assumption leads to contradict $q_{1} \sqsubseteq q_{2}$. If all the occurrences of $r$ in $q_{2}$ are positive (resp. negative), let $q_{1}^{c-}$ (resp. $q_{1}^{c+}$ ) be the complete query obtained from $q_{1}^{c-\{r\}}$ by adding solely negative (resp. positive) literals with relation $r$. Since $q_{1} \sqsubseteq q_{2}$ there is a homomorphism from $q_{2}$ to $q_{1}^{c-}$ (resp. $q_{1}^{c+}$ ). This homomorphism necessarily maps all occurrences of $r$ in $q_{2}$ into $q_{1}$; more generally, no literal of $q_{2}$ can be mapped to the added negative (resp. positive) occurrences of $r$. $h$ is thus a homomorphism from $q_{2}$ to $q_{1}^{c-\{r\}}$, which contradicts the hypothesis.

As a corollary to the previous properties, we obtain:

Theorem 1. $q_{1} \sqsubseteq q_{2}$ iff $q_{2}$ can be mapped to each completion of $q_{1}$ w.r.t. relations occurring in both positive and negative forms in $q_{1}$ and $q_{2}$.

Let us consider the queries in example 3: as $\neg s$ does not appear in $q_{1}$, it can be immediately concluded that $q_{1} \nsubseteq q_{2}$. Would $\neg s(w, w)$ not exist in $q_{2}$, as $r$ does not appear positively and negatively both in $q_{1}$ and $q_{2}$, no relation can be used for completion, thus there is also immediate failure.

\subsection{Special subgraphs}

As we have seen, a homomorphism from $q_{2}$ to $q_{1}$ is a sufficient but not necessary condition for containment. The objective here is to identify parts - or subgraphs - of $q_{2}$ (i.e. conjunctions of literals appearing in $q_{2}$ ) for which there must be a homomorphism to $q_{1}$. Moreover, such a homomorphism from a subgraph of $q_{2}$ to $q_{1}$ has to be potentially extensible to a homomorphism from the entire $q_{2}$ to a completion of $q_{1}$. We call it a compatible homomorphism. See Figure 3: there are three homomorphisms from $q_{2}^{-}$to $q_{1}: h_{1}=\{x \rightarrow t, y \rightarrow u, z \rightarrow w\}, h_{2}=\{x \rightarrow t, y \rightarrow w, z \rightarrow v\}$, 

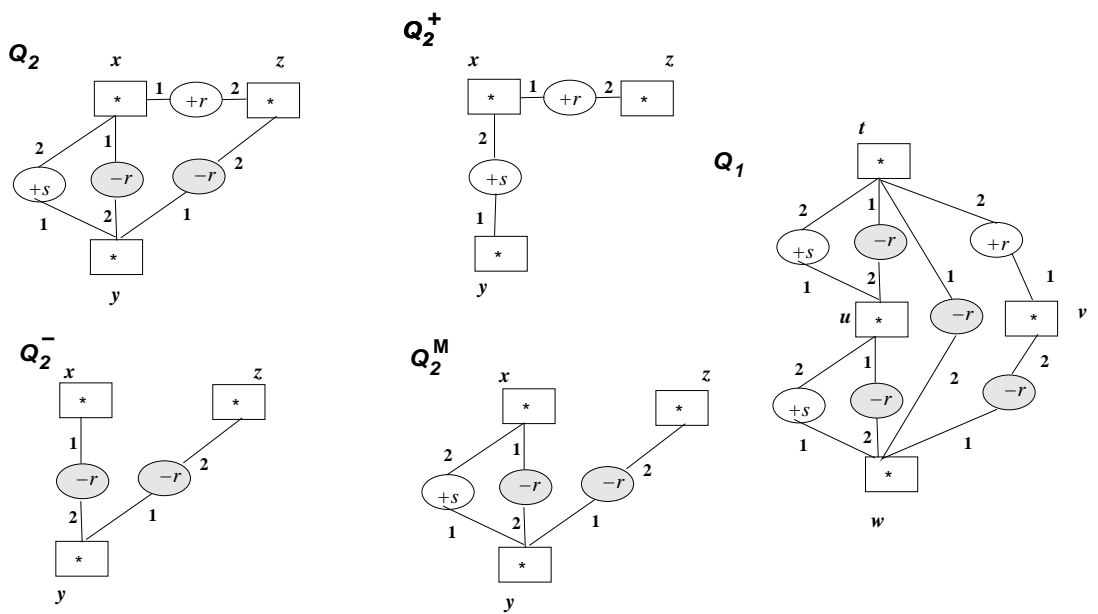

$q_{2}=\operatorname{ans}() \leftarrow s(y, x) \wedge r(x, z) \wedge \neg r(x, y) \wedge \neg r(y, z)$

$q_{1}=\operatorname{ans}() \leftarrow s(u, t) \wedge r(t, v) \wedge s(w, u) \wedge \neg r(u, w) \wedge \neg r(t, u) \wedge \neg r(w, v) \wedge \neg r(t, w)$

Fig. 3. Pure subgraphs of $q_{2}\left(q_{1} \sqsubseteq q_{2}\right)$

$h_{3}=\{x \rightarrow u, y \rightarrow w, z \rightarrow v\}$. To check the compatibility, we have to consider $s(y, x)$ and $r(x, z) . h_{1}$ is not compatible because it leads to map $r(x, z)$ to $\neg r(t, w)$.

Definition 2 (compatible homomorphism). Given two queries $q_{2}$ and $q_{1}$, and $q_{2}{ }_{2}$ any subgraph of $q_{2}$ defining a well-formed $P G$ (i.e. $q_{2}^{\prime}$ is any conjunction of literals appearing in $q_{2}$ ), a homomorphism $h$ from $q_{2}^{\prime}$ to $q_{1}$ is said to be compatible (w.r.t. $q_{2}$ ) if for each literal of $q_{2}$ that does not appear in $q_{2}^{\prime}$ but has all its terms in $q_{2}^{\prime}$, say $t_{1} \ldots t_{k}$, there is no literal with the same relation and the opposite polarity on $h\left(t_{1}\right) \ldots h\left(t_{k}\right)$ in $q_{1}$.

Given a query $q$, the positive subgraph of $q$, denoted by $q^{+}$is the subgraph obtained from $q$ by selecting only the positive literals of $q$. The negative subgraph $q^{-}$of $q$ is the dual notion, that is the subgraph obtained from $q$ by selecting only the negative literals of $q$. See $q_{2}^{+}$and $q_{2}^{-}$in Figure 3. The next property is the same as theorem 1 in [WL03] reformulated and proven in the graph framework, except that we extend the definition of $q^{+}$to non-safe queries. Note that, when $q$ is a safe query, $q^{+}$contains all term nodes of $q$.

Property 5. [WL03] If there is no compatible homomorphism from $q_{2}^{+}$to $q_{1}$ (or equivalently to $q_{1}^{+}$), then $q_{1} \nsubseteq q_{2}$.

Proof. Let $q_{1}^{c-}$ be the negative completion of $q_{1}$. If $q_{1} \sqsubseteq q_{2}$ then there is a homomorphism $h$ from $q_{2}$ to $q_{1}^{c-}$, which necessarily maps $q_{2}^{+}$to $q_{1}^{+}$. Let $l_{i}=\neg r\left(t_{1} \ldots t_{k}\right)$ be any negative literal of $q_{2}$. Since $h$ is a homomorphism, $q_{1}^{c-}$ contains a literal $\neg r\left(h\left(t_{1}\right) \ldots h\left(t_{k}\right)\right)$. As $q_{1}$ is consistent, it cannot contain a literal $r\left(h\left(t_{1}\right) \ldots h\left(t_{k}\right)\right)$. We conclude that $h$ with domain restricted to $q_{2}^{+}$is a compatible homomorphism to $q_{1}$.

A similar property is obtained by considering $q_{2}^{-}$instead of $q_{2}^{+}$. 
Property 6. If there is no compatible homomorphism from $q_{2}^{-}$to $q_{1}$ (or equivalently to $\left.q_{1}^{-}\right)$, then $q_{1} \nsubseteq q_{2}$.

Proof. Consider $q_{1}^{c+}$ the positive completion of $q_{1}$ instead of $q_{1}^{c-}$.

Both $q_{2}^{+}$and $q_{2}^{-}$notions can be generalized in the following way.

Definition 3 ( $q_{2}^{\max }$ pure subgraph). A pure subgraph of $q_{2}$ is a subgraph that does not contain opposite occurrences of the same relation. We note $q_{2}^{\max }$ a pure subgraph of $q_{2}$ that is maximal for inclusion.

Observe that a $q_{2}^{\max }$ is obtained from $q_{2}$ by selecting, for each relation appearing in $q_{2}$, either all its positive occurrences or all its negative occurrences. See Figure 3: $q_{2}$ has two pure subgraphs maximal for inclusion; $q_{2}^{+}$and $q_{2}^{M} \cdot q_{2}^{+}$(resp. $q_{2}^{-}$) is the particular case where positive (resp. negative) occurrences are chosen for all relations; but it is not necessarily maximal for inclusion as a relation may appear only negatively (res. positively). The ans $_{i}$ relation is a particular case of such relation.

Theorem 2. If there is a $q_{2}^{\max }$ that cannot be mapped by a compatible homomorphism to $q_{1}$, then $q_{1} \nsubseteq q_{2}$.

Proof. Consider $q_{1}^{-\max }$ as the completion of $q_{1}$ built as follows: for each relation $r$, if $r$ occurs positively (resp. negatively) in $q_{2}^{\max }$ then complete $q_{1}$ with negative (resp. positive) occurrences of $r$. If $q_{2}^{\max }$ cannot be mapped to $q_{1}$ by a compatible homomorphism, then it cannot be mapped by a compatible homomorphism to $q_{1}^{-\max }$ (since by construction no literal of $q_{2}^{\max }$ can be mapped to an added literal). Since $q_{2}$ cannot be mapped to $q_{1}^{-\max }, q_{1}^{-\max }$ is a counter-example to the property $q_{1} \sqsubseteq q_{2}$.

This theorem can be slightly extended by taking into account the occurrences of terms in the literals.

Definition 4. Two literals are said to be dependant if (1) they have an opposite polarity, (2) they have the same relation and (3) their atoms are unifiable after a renaming of their common variables. Otherwise they are said to be independant.

Two atoms are not unifiable after a renaming of their common variables if their unification would lead to unify different constants. For instance, let $a$ and $b$ be distinct constants; $r(x, a)$ and $\neg r(y, b)$ are independant literals; $p(x, x, a)$ and $\neg p(b, y, y)$ are independant literals as well, whereas $r(x, a)$ and $\neg r(b, y)$ are dependant literals.

Definition 5. An independant subgraph of a query $q_{2}$ is a subgraph of $q_{2}$ composed of pairwise independant literals.

More generally, let us say that two literals are "exchangeable" if they can have the same list of images for their arguments by homomorphisms to (necessarily distinct) completions of $q_{1}$. F.i. given the distinct constants $a$ and $b$, the literals $r(x, a)$ and $\neg r(b, y)$ are dependant but, if $q_{1}$ contains $r(a, b)$, they are not exchangeable. Independant subgraphs, and a fortiori pure subgraphs, are only particular cases of subgraphs without exchangeable literals; the general notion of "exchangeability" remains to be studied. Exchangeable literals are responsible for the problem complexity, as shown by the next property. 
Property 7. If $q_{1} \sqsubseteq q_{2}$, then there is a compatible homomorphism from every subgraph of $q_{2}$ composed of pairwise non-exchangeable literals to $q_{1}$.

Sketch of proof. Consider such a subgraph $q^{\prime}$ of $q_{2}$. Let $q_{1}^{c+}$ be the completion of $q_{1}$ with solely positive literals. If there is no homomorphism from $q^{\prime}$ to $q_{1}$, then for each homomorphism from $q^{\prime}$ to $q_{1}^{c+}$, there is at least one added literal, say $p(u)$, such that a literal $p(v)$ in $q^{\prime}$ is mapped to $p(u)$. Let us replace all such $p(u)$ by $\neg p(u)$. Let $q_{1}^{c^{\prime}}$ be the graph obtained. Let $h$ be a homomorphism from $q^{\prime}$ to $q_{1}^{c^{\prime}}$ (there is such a homomorphism since $\left.q_{1} \sqsubseteq q_{2}\right)$. $h$ maps a literal $\neg p(w)$ in $q^{\prime}$ to a literal $\neg p(u)$, otherwise there would be a homomorphism from $q^{\prime}$ to $q_{1}$. By construction, there is a literal $p(v)$ mapped to $p(u)$ by a homomorphism from $q^{\prime}$ to $q_{1}^{c+}$, thus $p(v)$ and $\neg p(w)$ are exchangeable literals.

The following extension to the theorem 2 is a corollary of property 7 .

Theorem 3. [Extension to the theorem 2] If there is an independant subgraph of $q_{2}$ that cannot be mapped by a compatible homomorphism to $q_{1}$, then $q_{1} \nsubseteq q_{2}$.

We thus obtain a case for which CQC has the same complexity as homomorphism checking:

Property 8. If $q_{2}$ is an independant subgraph, then $q_{1} \sqsubseteq q_{2}$ iff there is a homomorphism from $q_{2}$ to $q_{1}$.

\subsection{Filtering implementation}

Let us end this section with an implementation of some filtering properties (algorithm 2 that will be used next section).

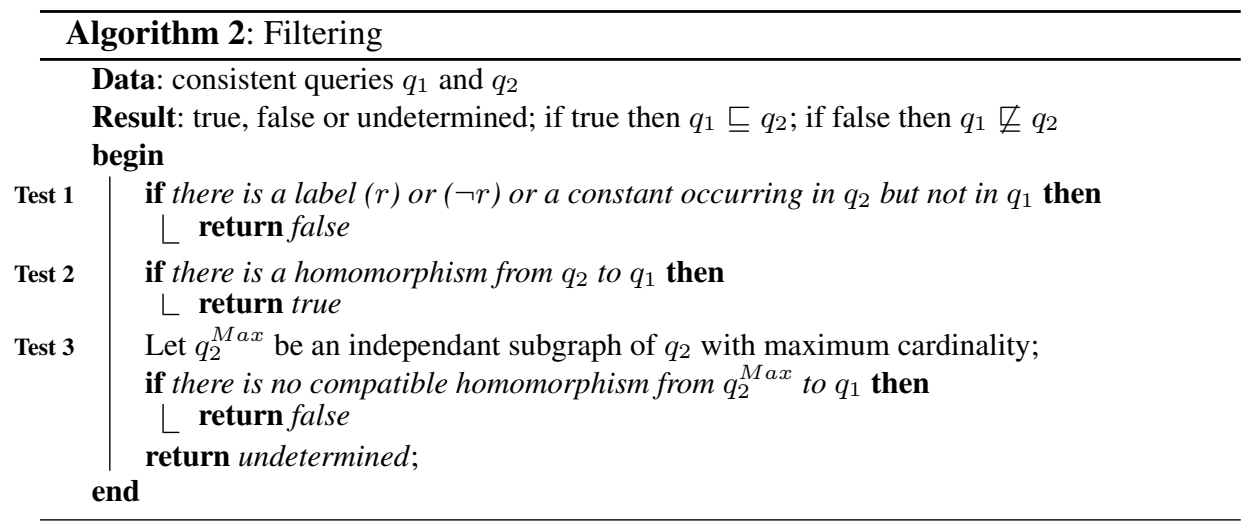

Roughly, Test 1 is in $\mathcal{O}\left(r \log _{2}(r)\right)$ where $r$ is the maximum number of relations in $q_{1}$ or $q_{2}$. Test 2 and Test 3 perform a homomorphism check. For Test 3, choosing a subgraph with maximum size is an obvious choice but there may be other criteria f.i. based on the structure of the obtained subgraph. Alternatively, one can choose to check several or all independant subgraphs instead of one. 


\section{Space exploration algorithm}

The space of queries "leading" from $q_{1}$ to its completions is structured in a sup-semilattice by graph inclusion (given two queries $q_{1}$ and $q_{2}$ in this space, $q_{1} \leq q_{2}$ if $q_{2}$ is a subgraph of $q_{1}$ ). The question "is there a homomorphism from $q_{2}$ to each $q_{1}^{c}$ (completion of $q_{1}$ )" can be reformulated as follows "is there a covering set, that is a subset of incomparable queries of this space $\left\{q_{1}, \ldots, q_{k}\right\}$ such that (1) there is a homomorphism from $q_{2}$ to each $q_{i} ;(2)$ for each $q_{1}^{c}$ there is a $q_{i}$ with $q_{1}^{c} \leq q_{i}$.

The brute-force algorithm (Algorithm 1) takes the set of all completions of $q_{1}$ as covering set. The next algorithm (Algorithm 3 and recursive search Algorithm 4) searches the space in a top-down way starting from $q_{1}$ and tries to build a covering set with partial completions of $q_{1}$. Case-based reasoning is applied at each step: for a given relation $r$ with arity $k$ and a tuple $\left(t_{1} \ldots t_{k}\right)$ such that neither $r\left(t_{1} \ldots t_{k}\right)$ nor $\neg r\left(t_{1} \ldots t_{k}\right)$ is present in the current partial completion, two queries are generated according to each case. The algorithm is justified by the following property:

Theorem 4. $q_{1} \sqsubseteq q_{2}$ if and only if:

1. There is a homomorphism hrom $q_{2}$ to $q_{1}$ or

2. $q^{\prime} \sqsubseteq q_{2}$ and $q^{\prime \prime} \sqsubseteq q_{2}$ where $q^{\prime}$ (resp. $q^{\prime \prime}$ ) is obtained from $q_{1}$ by adding the positive literal $r\left(t_{1} \ldots t_{k}\right)$ (resp. the negative literal $\neg r\left(t_{1} \ldots t_{k}\right)$ ) where $r$ is a relation of arity $k$ occurring in $q_{2}$ (both in positive and negative forms) and $t_{1} \ldots t_{k}$ are terms of $q_{1}$ such that neither the literal $r\left(t_{1} \ldots t_{k}\right)$ nor the literal $\neg r\left(t_{1} \ldots t_{k}\right)$ is already present in $q_{1}$.

Proof (sketch). $(\Rightarrow)$ By recurrence on the number of literals to add to $q_{1}$ to obtain a complete query. $(\Leftarrow)$ Condition 1 corresponds to property 1 . For condition 2 , see that $\left\{q^{\prime}, q^{\prime \prime}\right\}$ is a covering set.

Subalgorithm 4 is supposed to have direct access to data available in the main algorithm 3. The choice of $r$ and $t_{1} \ldots t_{k}$, in Algorithm 4, can be guided by a compatible homomorphism from an independant graph.

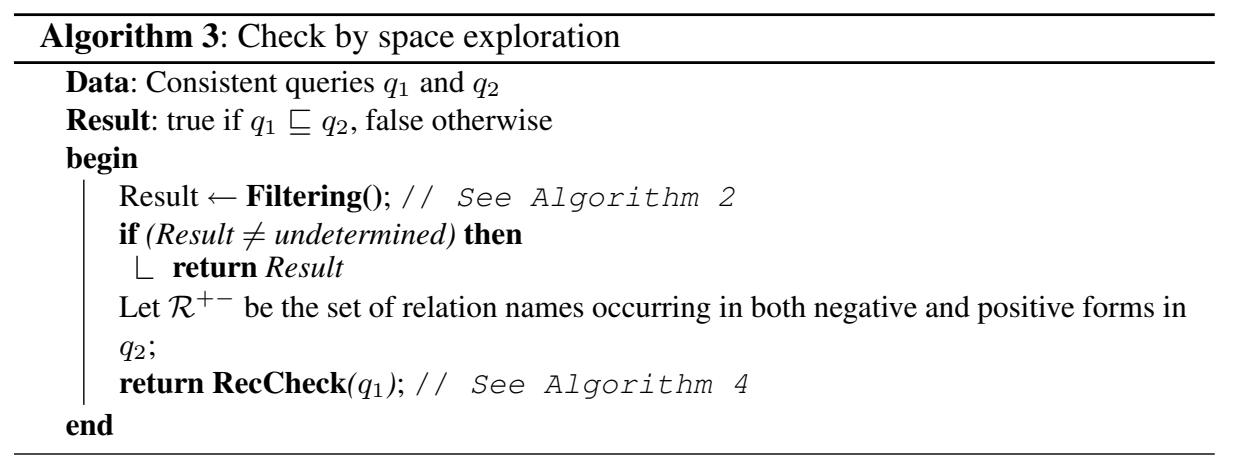

The following property ensures that Algorithm 4 does not generate the same query several times, which is a crucial point for complexity. Otherwise the algorithm could be worse than the brute-force algorithm in the worse-case. 


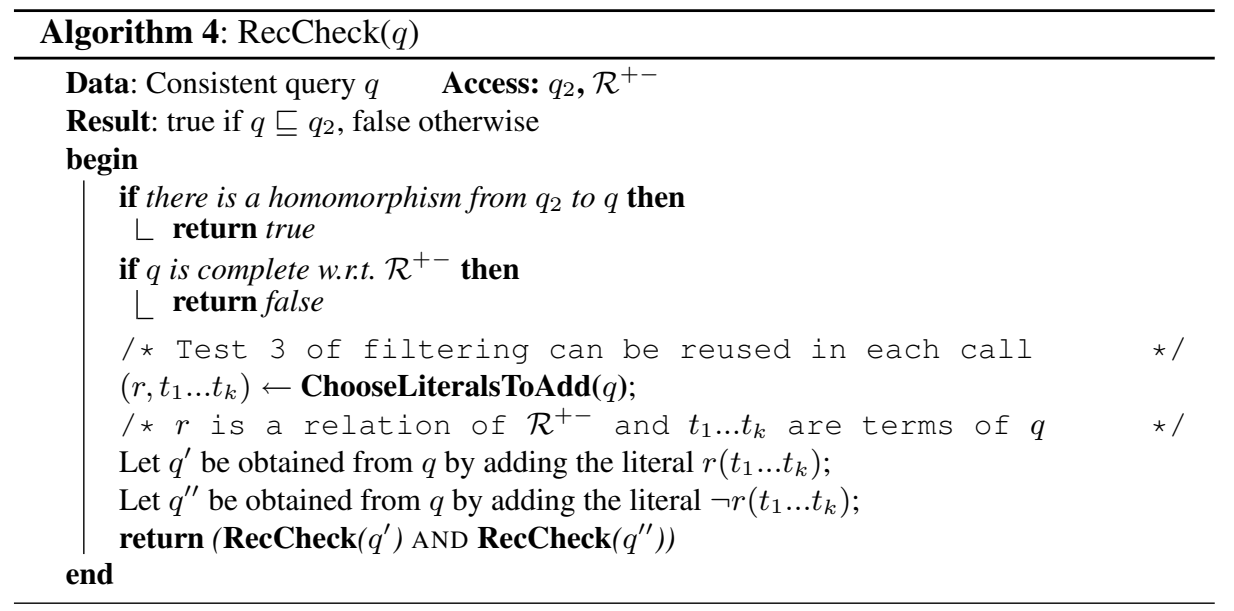

Property 9. The subspace explored by Algorithm 4 is a (binary) tree.

Indeed, at each recursive call, $\left\{q^{\prime}, q^{\prime \prime}\right\}$ is a covering set inducing a bipartition of the covered space: each query in this space is below exactly one of these two queries.

Property 10. Algorithm 3 has the same time and space complexity as Algorithm 1.

Proof. Property 9 ensures that the number of queries generated is at most twice the number of completions of $q_{1}$ (in the worse case, the complete queries are the leaves of the generated tree of queries). Checking whether a query is complete can be done in constant time if the number of literals in the query is incrementally maintained. Thus time complexity is the same as Algorithm 1. For space complexity, see that the tree is explored in a depth-first way.

Wei and Lausen's algorithm is based on the following theorem (theorem 2 of their paper reformulated in graph terms; moreover, in (1) "compatible" has been added, as well as step (2.1) to prevent inconsistent queries to be built ${ }^{3}$ ). This theorem considers safe queries (otherwise $h$ could be undefined on some variables in $q_{2}$ ).

Theorem 5. [WL03] With $q_{1}$ and $q_{2}$ being safe queries, $q_{1} \sqsubseteq q_{2}$ if and only if:

1. There is a compatible homomorphism hrom $q_{2}^{+}$to $q_{1}^{+}$, such that:

2. for each negative literal $l_{i}=\neg r\left(t_{1} \ldots t_{k}\right)$ in $q_{2}$, (2.1) either $h$ can be extended to include $l_{i}$ or (2.2) $q_{i}^{\prime} \sqsubseteq q_{2}$ holds, where $q_{i}^{\prime}$ is obtained from $q_{1}$ by adding the positive literal $r\left(h\left(t_{1}\right) \ldots h\left(t_{k}\right)\right)$.

Note that if each negative literal $l_{i}$ can be included in $h$ then $h$ is a homomorphism from $q_{2}$ in $q_{1}$. An important point is that this theorem induces a radically different way of searching the space than that of Algorithm 3. Indeed, whereas Algorithm 3 develops a tree, condition (2) leads to build a covering set that does not partition the space. An algorithm applying this property directly is thus likely to explore the same subspaces several times.

\footnotetext{
${ }^{3}$ Indeed, the theorem does not apply to inconsistent queries. If $q_{1}$ is inconsistent, it is by definition included in any $q_{2}$, but there might be no homomorphism from $q_{2}^{+}$to $q_{1}^{+}$.
} 
The algorithm proposed by Wei and Lausen (in the appendix of their paper) sketchily proceeds as follows. First, all homomorphisms from $q_{2}^{+}$to $q_{1}^{+}$are generated. Then for each compatible homomorphism, say $h$, and for each negative literal that cannot be mapped by extending $h$, a new query to test is generated from $q_{1}$ by adding a positive literal according to the previous theorem. This algorithm can be seen as developing a and/or tree: a homomorphism $h$ leads to success if all queries $q_{i}^{\prime}$ directly generated from it lead to containment; a query $q_{i}^{\prime}$ leads to containment if there is a homomorphism from $q_{i}^{\prime+}$ leading to success. The and/or tree is traversed in a breadth-first manner.

This algorithm has a space complexity exponential in the size of the initial queries, at least because all homomorphisms from $q_{2}^{+}$to $q_{1}^{+}$are first generated and the and/or tree is traversed in a breadth-first manner. Concerning time complexity, the key question is whether the same query can be generated several times. The notion of "new" mapping is mentioned in the algorithm (when the homomorphisms from $q_{2}^{+}$to $q_{1}^{+}$are enumerated, only new mappings are retained) but without detail about how a "new" mapping is recognized. A priori one has to store all already generated mappings to recognize a new one. If so, the space complexity would be exponential in the size of $q_{2}$ even with the assumption that homomorphisms are generated one by one and the tree is traversed in a depth-first way. To summarize, the algorithm we propose in this paper (see Algorithms 3 and 4) has the following qualities compared to Wei and Lausen's algorithm:

- it is not restricted to safe queries;

- the space exploration is based on special subgraphs, which generalize the $q_{2}^{+}$notion (and could be used instead of it in condition 1 of Wei and Lausen's theorem);

- it is polynomial in space (if the arity of relations is bound by a constant);

- it is simple to describe and implement.

Acknowledgments. We specially thank a referee for his/her valuable comments.

\section{References}

[ASU79] A. V. Aho, Y. Sagiv, and J. D. Ullman. Equivalences among relational expressions. SIAM J. Comput., 8(2):218-246, 1979.

[CM77] A.K. Chandra and P.M. Merlin. Optimal implementation of conjunctive queries in relational databases. In 9th ACM Symposium on Theory of Computing, pages 77-90, 1977.

[Hal01] A. Y. Halevy. Answering queries using views: A survey. VLDB Journal: Very Large Data Bases, 10(4):270-294, 2001.

[Ker01] G. Kerdiles. Saying it with Pictures: a Logical Landscape of Conceptual Graphs. PhD thesis, Univ. Montpellier II / Amsterdam, Nov. 2001.

[LS93] A. Y. Levy and Y. Sagiv. Queries independent of updates. In $V L D B$, pages 171-181, 1993.

[ML06] M.L. Mugnier and M. Leclère. On querying simple conceptual graphs with negation. Data and Knowledge Engineering (DKE), 2006. In press, doi:10.1016/j.datak.2006.03.008.

[U1189] J. D. Ullman. Principles of Database and Knowledge-Base Systems, Volume II. Computer Science Press, 1989.

[U1197] J. D. Ullman. Information Integration using Logical Views. In International Conference on Database Theory (ICDT), 1997.

[WL03] F. Wei and G. Lausen. Containment of Conjunctive Queries with Safe Negation. In International Conference on Database Theory (ICDT), 2003. 